\title{
Many-to-English Machine Translation Tools, Data, and Pretrained Models
}

\author{
Thamme Gowda \\ Information Sciences Institute \\ University of Southern California \\ tg@isi.edu
}

\section{Chris A Mattmann}

NASA Jet Propulsion Laboratory

California Institute of Technology

chris.a.mattmann@jpl.nasa.gov

\begin{abstract}
While there are more than 7000 languages in the world, most translation research efforts have targeted a few high resource languages. Commercial translation systems support only one hundred languages or fewer, and do not make these models available for transfer to low resource languages. In this work, we present useful tools for machine translation research: MTDATA, NLCODEC, and RTG. We demonstrate their usefulness by creating a multilingual neural machine translation model capable of translating from 500 source languages to English. We make this multilingual model readily downloadable and usable as a service, or as a parent model for transfer-learning to even lower-resource languages. ${ }^{1}$
\end{abstract}

\section{Introduction}

Neural machine translation (NMT) (Bahdanau et al., 2015; Vaswani et al., 2017) has progressed to reach human performance on select benchmark tasks (Barrault et al., 2019, 2020). However, as MT research has mainly focused on translation between a small number of high resource languages, the unavailability of usable-quality translation models for low resource languages remains an ongoing concern. Even those commercial translation services attempting to broaden their language coverage has only reached around one hundred languages; this excludes most of the thousands of languages used around the world today.

Freely available corpora of parallel data for many languages are available, though they are hosted at various sites, and are in various forms. A challenge for incorporating more languages into MT models is a lack of easy access to all of these datasets. While standards like ISO 639-3 have been established to

\footnotetext{
${ }^{1}$ Demo website: http://rtg.isi.edu/many-eng. Video demo: https : //youtu . be/NSYO-Mv01KE.
}

\author{
Zhao Zhang \\ Texas Advanced Computing Center \\ University of Texas \\ zzhang@tacc.utexas.edu \\ Jonathan May \\ Information Sciences Institute \\ University of Southern California \\ jonmay@isi.edu
}

bring consistency to the labeling of language resources, these are not yet widely adopted. In addition, scaling experimentation to several hundred languages on large corpora involves a significant engineering effort. Simple tasks such as dataset preparation, vocabulary creation, transformation of sentences into sequences, and training data selection becomes formidable at scale due to corpus size and heterogeneity of data sources and file formats. We have developed tools to precisely address all these challenges, which we demonstrate in this work.

Specifically, we offer three tools which can be used either independently or in combination to advance NMT research on a wider set of languages (Section 2): firstly, MTDATA, which helps to easily obtain parallel datasets (Section 2.1); secondly, NLCODEC, a vocabulary manager and storage layer for transforming sentences to integer sequences, that is efficient and scalable (Section 2.2); and lastly, RTG, a feature-rich Pytorch-backed NMT toolkit that supports reproducible experiments (Section 2.3).

We demonstrate the capabilities of our tools by preparing a massive bitext dataset with more than 9 billion tokens per side, and training a single multilingual NMT model capable of translating 500 source languages to English (Section 3). We show that the multilingual model is usable either as a service for translating several hundred languages to English (Section 4.1), or as a parent model in a transfer learning setting for improving translation of low resource languages (Section 4.2).

\section{Tools}

Our tools are organized into the following sections:

\subsection{MTDATA}

MTDATA addresses an important yet often overlooked challenge - dataset preparation. By assign- 


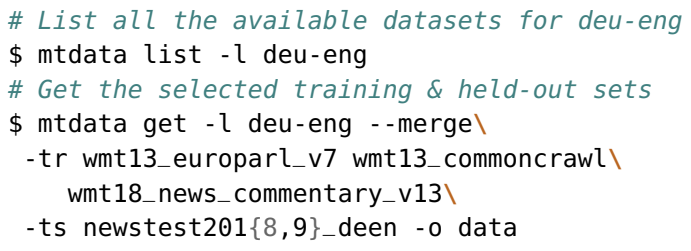

Listing 1: MTDATA examples for listing and downloading German-English datasets. The -merge flag results in merging all of the training datasets specified by - $t r$ argument into a single file.

ing an ID for datasets, we establish a clear way of communicating the exact datasets used for MT experiments, which helps in reproducing the experimental setup. By offering a unified interface to datasets from many heterogeneous sources, MTDATA hides mundane tasks such as locating URLs, downloading, decompression, parsing, and sanity checking. Some noteworthy features are:

- Indexer: a large index of publicly available parallel datasets.

- Normalizer: maps language names to ISO-639-3 codes which has representation space for $7800+$ languages. ${ }^{2}$

- Parsers: parses heterogeneous data formats for parallel datasets, and produces a simple plain text file by merging all the selected datasets.

- Extensible: new datasets and parsers can be easily added.

- Local Cache: reduces network transfers by maintaining a local cache, which is shared between experiments.

- Sanity Checker: performs basic sanity checks such as segment count matching and empty segment removal. When error states are detected, stops the setup with useful error messages.

- Reproducible: stores a signature file that can be used to recreate the dataset at a later time.

- Courtesy: shows the original BIBTEX citation attributed to datasets.

- Easy Setup: pip install mtdata

- Open-source:

https://github.com/thammegowda/mtdata

Listing 1 shows an example for listing and getting datasets for German-English. In Section 3.1, we use MTDATA ${ }^{3}$ to obtain thousands of publicly available datasets for a large many-to-English translation experiment.

\footnotetext{
${ }^{2}$ https://iso639-3.sil.org

${ }^{3}$ At the time of writing, v0.2.8
}

\subsection{NLCODEC}

NLCODEC is a vocabulary manager with encodingdecoding schemes to transform natural language sentences to and from integer sequences.

\section{Features:}

- Versatile: Supports commonly used vocabulary schemes such as characters, words, and bytepair-encoding (BPE) subwords (Sennrich et al., 2016).

- Scalable: Apache $\operatorname{Spark}^{4}$ (Zaharia et al., 2016) backend can be optionally used to create vocabulary from massive datasets.

- Easy Setup: pip install nlcodec

- Open-source:

https://github. com/isi-nlp/nlcodec/

When the training datasets are too big to be kept in the primary random access memory (RAM), the use of secondary storage is inevitable. The training processes requiring random examples lead to random access from a secondary storage device. Even though the latest advancements in secondary storage technology such as solid-state drive (SSD) have faster serial reads and writes, their random access speeds are significantly lower than that of RAM. To address these problems, we include an efficient storage and retrieval layer, NLDB, which has the following features:

- Memory efficient by adapting datatypes based on vocabulary size. For instance, encoding with vocabulary size less than 256 (such as characters) can be efficiently represented using 1-byte unsigned integers. Vocabularies with fewer than 65,536 types, such as might be generated when using subword models (Sennrich et al., 2016) require only 2-byte unsigned integers, and 4-byte unsigned integers are sufficient for vocabularies up to 4 billion types. As the default implementation of Python, CPython, uses 28 bytes for all integers, we accomplish this using NumPy (Harris et al., 2020). This optimization makes it possible to hold a large chunk of training data in smaller RAM, enabling a fast random access.

- Parallelizable: Offers a multi-part database by horizontal sharding that supports parallel writes (e.g., Apache Spark) and parallel reads (e.g., distributed training).

- Supports commonly used batching mechanisms such as random batches with approximatelyequal-length sequences.

NLDB has a minimal footprint and is part of

\footnotetext{
${ }^{4}$ https://spark. apache.org/
} 
the NLCODEC package. In Section 3, we take advantage of the scalability and efficiency aspects of NLCODEC and NLDB to process a large parallel dataset with 9 billion tokens on each side.

\subsection{RTG}

Reader translator generator (RTG) is a neural machine translation (NMT) toolkit based on Pytorch (Paszke et al., 2019). Notable features of RTG are:

- Reproducible: All the required parameters of an experiment are included in a single YAML configuration file, which can be easily stored in a version control system such as git or shared with collaborators.

- Implements Transformer (Vaswani et al., 2017), and recurrent neural networks (RNN) with crossattention models (Bahdanau et al., 2015; Luong et al., 2015).

- Supports distributed training on multi-node multi-GPUs, gradient accumulation, and Float16 operations.

- Integrated Tensorboard helps in visualizing training and validation losses.

- Supports weight sharing (Press and Wolf, 2017), parent-child transfer (Zoph et al., 2016), beam decoding with length normalization (Wu et al., 2016), early stopping, and checkpoint averaging.

- Flexible vocabulary options with NLCODEC and SentencePiece (Kudo and Richardson, 2018) which can be either shared or separated between source and target languages.

- Easy setup: pip install rtg

- Open-source: https://isi-nlp.github.io/rtg/

\section{Many-to-English Multilingual NMT}

In this section, we demonstrate the use of our tools by creating a massively multilingual NMT model from publicly available datasets.

\subsection{Dataset}

We use MTDATA to download datasets from various sources, given in Table 1. To minimize data imbalance, we select only a subset of the datasets available for high resource languages, and select all available datasets for low resource languages. The selection is aimed to increase the diversity of data domains and quality of alignments.

Cleaning: We use SACREMOSES $^{5}$ to normalize

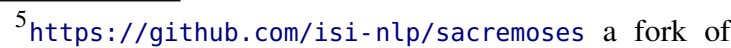
https://github. com/alvations/sacremoses with improvements to tokenization for many low resource languages.
}

\begin{tabular}{ll} 
Dataset & Reference \\
\hline \hline Europarl & Koehn (2005) \\
KFTT Ja-En & Neubig (2011) \\
Indian6 & Post et al. (2012) \\
OPUS & Tiedemann (2012) \\
UNPCv1 & Ziemski et al. (2016) \\
Tilde MODEL & Rozis and Skadinš (2017) \\
TEDTalks & Qi et al. (2018) \\
IITB Hi-En & Kunchukuttan et al. (2018) \\
Paracrawl & Esplà et al. (2019) \\
WikiMatrix & Schwenk et al. (2019) \\
JW300 & Agić and Vulić (2019) \\
PMIndia & Haddow and Kirefu (2020) \\
OPUS100 & Zhang et al. (2020) \\
WMT [13-20] & Bojar et al. (2013, 2014, 2015, 2016, \\
& 2017, 2018); Barrault et al. (2019, \\
& 2020)
\end{tabular}

Table 1: Various sources of MT datasets.

Unicode punctuations and digits, followed by word tokenization. We remove records that are duplicates, have abnormal source-to-target length ratios, have many non-ASCII characters on the English side, have a URL, or which overlap exactly, either on the source or target side, with any sentences in held out sets. As preprocessing is computeintensive, we parallelize using Apache Spark. The cleaning and tokenization results in a corpus of 474 million sentences and 9 billion tokens on the source and English sides each. The token and sentence count for each language are provided in Figure 1. Both the processed and raw datasets are available at http://rtg.isi.edu/many-eng/data/v1/. 6

\subsection{Many-to-English Multilingual Model}

We use RTG to train Transformer NMT (Vaswani et al., 2017) with a few modifications. Firstly, instead of a shared BPE vocabulary for both source and target, we use two separate BPE vocabularies. Since the source side has 500 languages but the target side has English only, we use a large source vocabulary and a relatively smaller target vocabulary. A larger target vocabulary leads to higher time and memory complexity, whereas a large source vocabulary increases only the memory complexity but not the time complexity. We train several models, ranging from the standard 6 layers, 512dimensional Transformers to larger ones with more parameters. Since the dataset is massive, a larger model trained on big mini-batches yields the best results. Our best performing model is a 768 dimensional model with 12 attention heads, 9 encoder layers, 6 decoder layers, feed-forward dimension of 2048, dropout and label smoothing at 0.1, using

\footnotetext{
${ }^{6}$ A copy is at https://opus.nlpl.eu/MT560.php
} 

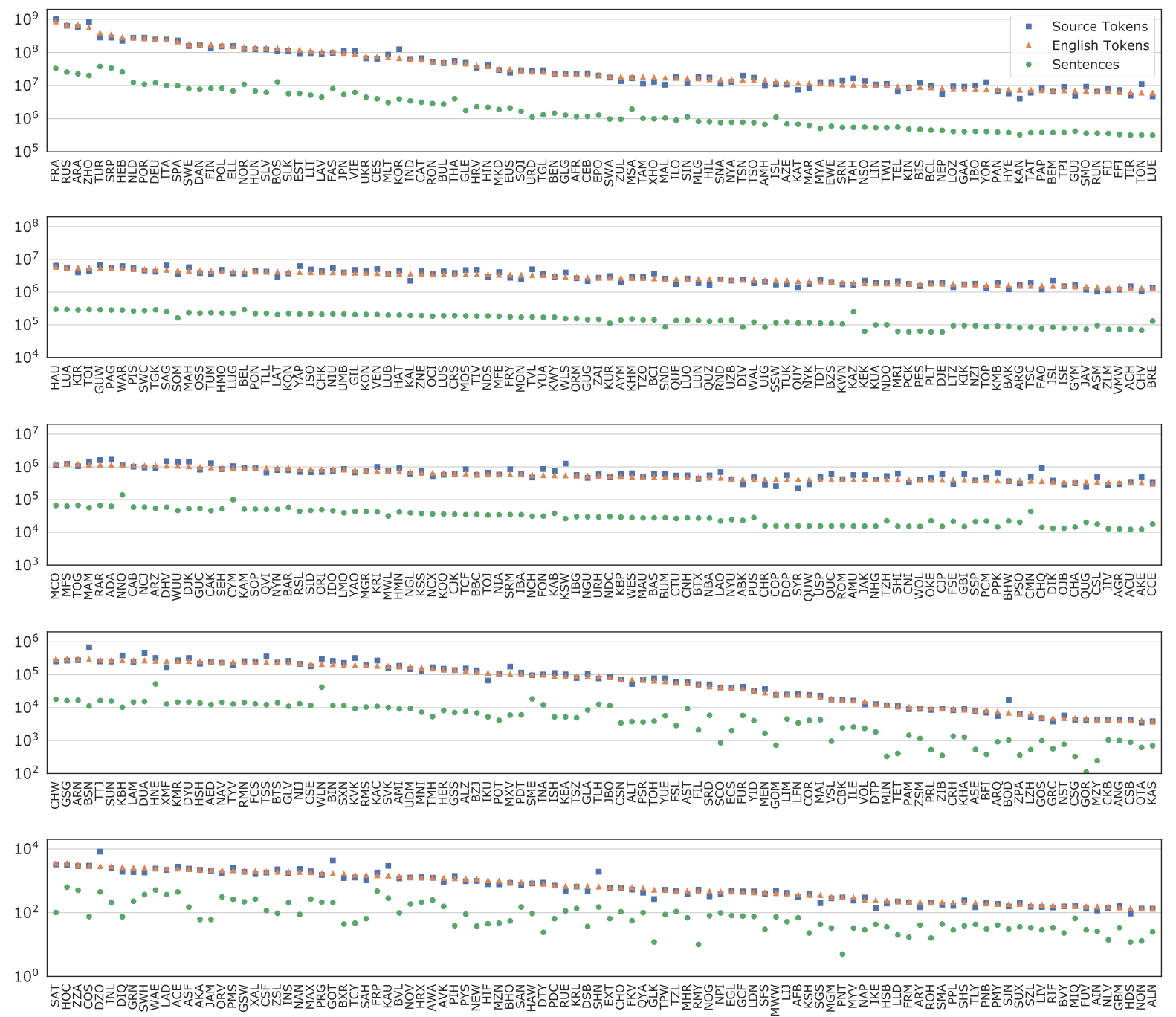

Figure 1: Training data statistics for the 500 languages, sorted based on descending order of English token count. These statistics are obtained after de-duplication and filtering (see Section 3.1). The full name for these ISO 639-3 codes can be looked up using MTDATA, e.g. mtdata-iso eng .

512,000 and 64,000 BPE types as source and target vocabularies, respectively. The decoder's input and output embeddings are shared. Since some of the English sentences are replicated to align with many sentences from different languages (e.g. the Bible corpus), BPE merges are learned from the deduplicated sentences using NLCODEC. Our best performing model is trained with an effective batch size of about 720,000 tokens per optimizer step. Such big batches are achieved by using mixed-precision distributed training on 8 NVIDIA A100 GPUs with gradient accumulation of 5 minibatches, each having a maximum of 18,000 tokens. We use the Adam optimizer (Kingma and Ba, 2014) with 8000 warm-up steps followed by a decaying learning rate, similar to Vaswani et al. (2017). We stop training after five days and six hours when a total of $200 \mathrm{~K}$ updates are made by the optimizer; validation loss is still decreasing at this point. To assess the translation quality of our model, we report BLEU (Papineni et al., 2002; Post, 2018) ${ }^{7}$ on a subset of languages for which known test sets are available, as given in Figure 2, along with a comparison to Zhang et al. (2020)'s best model. ${ }^{8}$

\section{Applications}

The model we trained as a demonstration for our tools is useful on its own, as described in the following sections.

\footnotetext{
${ }^{7}$ All our BLEU scores are obtained from SACREBLEU BLEU+c. mixed+\#.1+s. exp+tok. 13a+v.1.4.13.

${ }^{8}$ Scores are obtained from https://github.com/ bzhangGo/zero/tree/master/docs/multilingual_laln_ lalt; accessed: 2021/03/30
} 


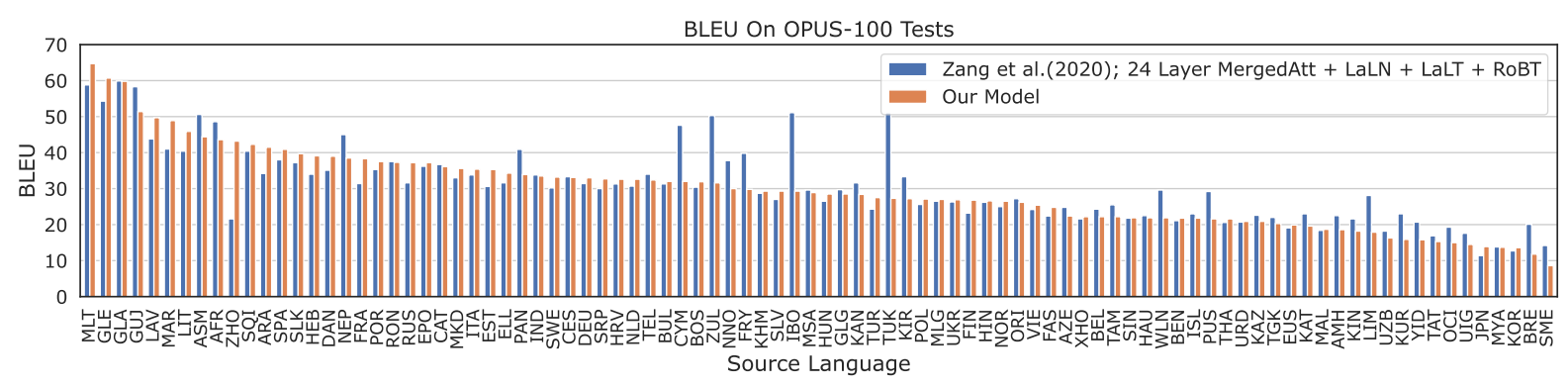

Figure 2: Many-to-English BLEU on OPUS-100 tests (Zhang et al., 2020). Despite having four times more languages on the source side, our model scores competitive BLEU on most languages with the strongest system of Zhang et al. (2020). The tests where our model scores lower BLEU have shorter source sentences (mean length of about three tokens).

\subsection{Readily Usable Translation Service}

Our pretrained NMT model is readily usable as a service capable of translating several hundred source languages to English. By design, source language identification is not necessary. Figure 2 shows that the model scores more than 20 BLEU, which maybe be a useful quality for certain downstream applications involving web and social media content analysis. Apache Tika (Mattmann and Zitting, 2011), a content detection and analysis toolkit capable of parsing thousands of file formats, has an option for translating any document into English using our multilingual NMT model. ${ }^{9}$ Our model has been packaged and published to DockerHub, ${ }^{10}$ which can be obtained by the following command:

IMAGE=tgowda/rtg-model : 500toEng $-\mathrm{v} 1$

docker run --rm -i -p 6060:6060 \$IMAGE

\# For GPU support: --gpus ' "device $=0$ " '

The above command starts a docker image with HTTP server having a web interface, as can be seen in Figure 3, and a REST API. An example interaction with the REST API is as follows:

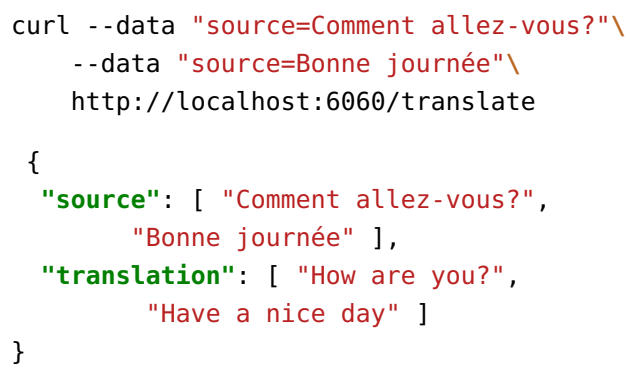

\subsection{Parent Model for Low Resource MT}

Fine tuning is a useful transfer learning technique for improving the translation of low resource languages (Zoph et al., 2016; Neubig and $\mathrm{Hu}, 2018$;

\footnotetext{
${ }^{9}$ https : //cwiki.apache. org/confluence/display/ TIKA/NMT - RTG

10 https: //hub. docker.com/
}

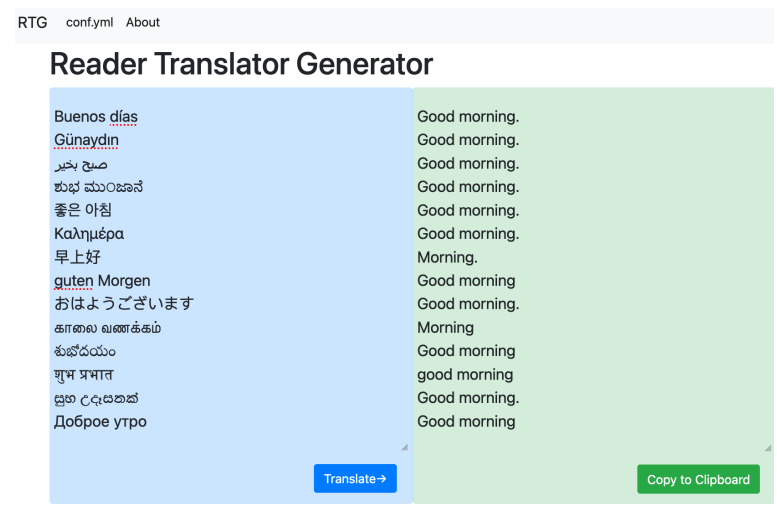

Figure 3: RTG Web Interface

Gheini and May, 2019). For instance, consider Breton-English (BRE-ENG) and Northern SamiEnglish (SME-ENG), two of the low resource settings for which our model has relatively poor BLEU (see Figure 2). To show the utility of fine tuning with our model, we train a strong baseline Transformer model, one for each language, from scratch using OPUS-100 training data (Zhang et al., 2020), and finetune our multilingual model on the same dataset as the baselines. We shrink the parent model vocabulary and embeddings to the child model dataset, and train all models on NVIDIA P100 GPUs until convergence. ${ }^{11}$ Table 2, which shows BLEU on the OPUS-100 test set for the two low resource languages indicates that our multilingual NMT parent model can be further improved with finetuning on limited training data. The finetuned model is significantly better than baseline model.

\footnotetext{
${ }^{11}$ More info: https://github.com/thammegowda/ 006 - many - to- eng/tree/master/lowres - xfer
} 


\begin{tabular}{lrr} 
Model & BRE-ENG & SME-ENG \\
\hline \hline Baseline & 12.7 & 10.7 \\
Parent & 11.8 & 8.6 \\
Finetuned & $\mathbf{2 2 . 8}$ & $\mathbf{1 9 . 1}$
\end{tabular}

Table 2: Finetuning our multilingual NMT on limited training data in low resource settings significantly improves translation quality, as quantified by BLEU.

\section{Related work}

\subsection{Tools}

SACREBLEU (Post, 2018) simplifies MT evaluation. MTDATA attempts to simplify training setup by automating training and validation dataset retrieval. OPUSTOOLs (Aulamo et al., 2020) is a similar tool however, it interfaces with OPUS servers only. Since the dataset index for OPUSTools is on a server, the addition of new datasets requires privileged access. In contrast, MTDATA is a client side library, it can be easily forked and extended to include new datasets without needing special privileges.

NLCODEC: NLCODEC is a Python library for vocabulary management. It overcomes the multithreading bottleneck in Python by using PySpark. SentencePiece (Kudo and Richardson, 2018) and HuggingfaceTokenizers (Wolf et al., 2020) are the closest alternatives in terms of features, however, modification is relatively difficult for Python users as these libraries are implemented in $\mathrm{C}++$ and Rust, respectively. In addition, SentencePiece uses a binary format for model persistence in favor of efficiency, which takes away the inspectability of the model state. Retaining the ability to inspect models and modify core functionality is beneficial for further improving encoding schemes, e.g. subword regularization (Kudo, 2018), BPE dropout (Provilkov et al., 2020), and optimal stop condition for subword merges (Gowda and May, 2020). FastBPE is another efficient BPE tool written in $\mathrm{C}++.{ }^{12}$ Subword-nmt (Sennrich et al., 2016) is a Python implementation of BPE, and stores the model in an inspectable plain text format, however, it is not readily scalable to massive datasets such as the one used in this work. None of these tools have an equivalent to NLDB's mechanism for efficiently storing and retrieving variable length sequences for distributed training.

RTG: Tensor2Tensor (Vaswani et al., 2018) originally offered the Transformer (Vaswani et al., 2017) implementation using Tensorflow (Abadi

\footnotetext{
12 https://github. com/glample/fastBPE
}

et al., 2015); our implementation uses Pytorch (Paszke et al., 2019) following Annotated Transformer (Rush, 2018). OpenNMT currently offers separate implementations for both Pytorch and Tensorflow backends (Klein et al., 2017, 2020). As open-source toolkits evolve, many good features tend to propagate between them, leading to varying degrees of similarities. Some of the available NMT toolkits are: Nematus (Sennrich et al., 2017), xNMT (Neubig et al., 2018). Marian NMT (Junczys-Dowmunt et al., 2018), Joey NMT (Kreutzer et al., 2019), Fairseq (Ott et al., 2019), and Sockey (Hieber et al., 2020). An exhaustive comparison of these NMT toolkits is beyond the scope of our current work.

\subsection{Multilingual NMT}

Johnson et al. (2017) show that NMT models are capable of multilingual translation without any architectural changes, and observe that when languages with abundant data are mixed with low resource languages, the translation quality of low resource pairs are significantly improved. They use a private dataset of 12 language pairs; we use publicly available datasets for up to 500 languages. Qi et al. (2018) assemble a multi-parallel dataset for 58 languages from TEDTalks domains, which are included in our dataset. Zhang et al. (2020) curate OPUS-100, a multilingual dataset of 100 languages sampled from OPUS, including test sets; which are used in this work. Tiedemann (2020) have established a benchmark task for 500 languages including single directional baseline models. Wang et al. (2020) examine the language-wise imbalance problem in multilingual datasets and propose a method to address the imbalance using a scoring function, which we plan to explore in the future.

\section{Conclusion}

We have introduced our tools: MTDATA for downloading datasets, NLCODEC for processing, storing and retrieving large scale training data, and RTG for training NMT models. Using these tools, we have collected a massive dataset and trained a multilingual model for many-to-English translation. We have demonstrated that our model can be used independently as a translation service, and also showed its use as a parent model for improving low resource language translation. All the described tools, used datasets, and trained models are made available to the public for free. 


\section{Acknowledgments}

The authors would like to thank Lukas Ferrer, Luke Miles, and Mozhdeh Gheini for their contributions to some of the tools used in this work, and thank Jörg Tiedemann for hosting our prepared dataset at OPUS (https://opus.nlpl.eu/MT560.php). The authors acknowledge the Center for Advanced Research Computing (CARC) at the University of Southern California for providing computing resources that have contributed to the research results reported within this publication. URL: https://carc.usc.edu. The authors acknowledge the Texas Advanced Computing Center (TACC) at The University of Texas at Austin for providing HPC resources that have contributed to the research results reported within this paper. URL: http://www.tacc.utexas.edu. This research is based upon work supported by the Office of the Director of National Intelligence (ODNI), Intelligence Advanced Research Projects Activity (IARPA), via AFRL Contract FA8650-17-C-9116. The views and conclusions contained herein are those of the authors and should not be interpreted as necessarily representing the official policies or endorsements, either expressed or implied, of the ODNI, IARPA, or the U.S. Government. The U.S. Government is authorized to reproduce and distribute reprints for Governmental purposes notwithstanding any copyright annotation thereon.

\section{Ethical Consideration}

Failure Modes: MTDATA will fail to operate, unless patched, when hosting services change their URLs or formats over time. On certain scenarios when a dataset has been previously accessed and retained in local cache, MTDATA continues to operate with a copy of previous version and ignores server side updates. We have done our best effort in normalizing languages to ISO 639-3 standard; our current version does not accommodate country and script variations of languages; e.g. UK English and US English are both mapped to eng. Our multilingual NMT model is trained to translate a full sentence at a time without considering source language information; translation of short phrases without a proper context might result in a poor quality translation.

Diversity and Fairness: We cover all languages on the source side for which publicly available dataset exists, which happens to be about 500 source languages. Our model translates to English only, hence only English speakers are benefited from this work.

Climate Impact: MTDATA reduces network transfers to the minimal by maintaining a local cache to avoid repetitive downloads. In addition to the raw datasets, preprocessed data is also available to avoid repetitive computation. Our Multilingual NMT has higher energy cost than a typical single directional NMT model due to higher number of parameters, however, since our single model translates hundreds of languages, the energy requirement is significantly lower than the total consumption of all independent models. Our trained models with all the weights are also made available for download.

Dataset Ownership: MTDATA is a client side library that does not have the ownership of datasets in its index. Addition, removal, or modification in its index is to be submitted by creating an issue at https://github.com/thammegowda/mtdata/issues. We ask the dataset users to review the dataset license, and acknowledge its original creators by citing their work, whose BIBTEX entries may be accessed using:

mtdata list - $\mathrm{n}$ <NAME $>$ - $\mathrm{l}<\mathrm{L} 1$ - L2 $>$ - full

The prepared dataset that we have made available for download includes citations.bib that acknowledges all the original creators of datasets. We do not vouch for quality and fairness of all the datasets.

\section{References}

Martín Abadi, Ashish Agarwal, Paul Barham, Eugene Brevdo, Zhifeng Chen, Craig Citro, Greg S. Corrado, Andy Davis, Jeffrey Dean, Matthieu Devin, Sanjay Ghemawat, Ian Goodfellow, Andrew Harp, Geoffrey Irving, Michael Isard, Yangqing Jia, Rafal Jozefowicz, Lukasz Kaiser, Manjunath Kudlur, Josh Levenberg, Dandelion Mané, Rajat Monga, Sherry Moore, Derek Murray, Chris Olah, Mike Schuster, Jonathon Shlens, Benoit Steiner, Ilya Sutskever, Kunal Talwar, Paul Tucker, Vincent Vanhoucke, Vijay Vasudevan, Fernanda Viégas, Oriol Vinyals, Pete Warden, Martin Wattenberg, Martin Wicke, Yuan Yu, and Xiaoqiang Zheng. 2015. TensorFlow: Large-scale machine learning on heterogeneous systems. Software available from tensorflow.org.

Željko Agić and Ivan Vulić. 2019. JW300: A widecoverage parallel corpus for low-resource languages. In Proceedings of the 57th Annual Meeting of the Association for Computational Linguistics, pages 3204-3210, Florence, Italy. Association for Computational Linguistics. 
Mikko Aulamo, Umut Sulubacak, Sami Virpioja, and Jörg Tiedemann. 2020. OpusTools and parallel corpus diagnostics. In Proceedings of the 12th Language Resources and Evaluation Conference, pages 3782-3789, Marseille, France. European Language Resources Association.

Dzmitry Bahdanau, Kyunghyun Cho, and Yoshua Bengio. 2015. Neural machine translation by jointly learning to align and translate. In 3rd International Conference on Learning Representations, ICLR 2015, San Diego, CA, USA, May 7-9, 2015, Conference Track Proceedings.

Loïc Barrault, Magdalena Biesialska, Ondřej Bojar, Marta R. Costa-jussà, Christian Federmann, Yvette Graham, Roman Grundkiewicz, Barry Haddow, Matthias Huck, Eric Joanis, Tom Kocmi, Philipp Koehn, Chi-kiu Lo, Nikola Ljubešić, Christof Monz, Makoto Morishita, Masaaki Nagata, Toshiaki Nakazawa, Santanu Pal, Matt Post, and Marcos Zampieri. 2020. Findings of the 2020 conference on machine translation (WMT20). In Proceedings of the Fifth Conference on Machine Translation, pages 1-55, Online. Association for Computational Linguistics.

Loïc Barrault, Ondřej Bojar, Marta R. Costa-jussà, Christian Federmann, Mark Fishel, Yvette Graham, Barry Haddow, Matthias Huck, Philipp Koehn, Shervin Malmasi, Christof Monz, Mathias Müller, Santanu Pal, Matt Post, and Marcos Zampieri. 2019. Findings of the 2019 conference on machine translation (WMT19). In Proceedings of the Fourth Conference on Machine Translation (Volume 2: Shared Task Papers, Day 1), pages 1-61, Florence, Italy. Association for Computational Linguistics.

Ondřej Bojar, Christian Buck, Chris Callison-Burch, Christian Federmann, Barry Haddow, Philipp Koehn, Christof Monz, Matt Post, Radu Soricut, and Lucia Specia. 2013. Findings of the 2013 Workshop on Statistical Machine Translation. In Proceedings of the Eighth Workshop on Statistical Machine Translation, pages 1-44, Sofia, Bulgaria. Association for Computational Linguistics.

Ondřej Bojar, Christian Buck, Christian Federmann, Barry Haddow, Philipp Koehn, Johannes Leveling, Christof Monz, Pavel Pecina, Matt Post, Herve Saint-Amand, Radu Soricut, Lucia Specia, and Aleš Tamchyna. 2014. Findings of the 2014 workshop on statistical machine translation. In Proceedings of the Ninth Workshop on Statistical Machine Translation, pages 12-58, Baltimore, Maryland, USA. Association for Computational Linguistics.

Ondřej Bojar, Rajen Chatterjee, Christian Federmann, Yvette Graham, Barry Haddow, Shujian Huang, Matthias Huck, Philipp Koehn, Qun Liu, Varvara Logacheva, Christof Monz, Matteo Negri, Matt Post, Raphael Rubino, Lucia Specia, and Marco Turchi. 2017. Findings of the 2017 conference on machine translation (WMT17). In Proceedings of the Second Conference on Machine Translation, pages 169214, Copenhagen, Denmark. Association for Computational Linguistics.

Ondřej Bojar, Rajen Chatterjee, Christian Federmann, Yvette Graham, Barry Haddow, Matthias Huck, Antonio Jimeno Yepes, Philipp Koehn, Varvara Logacheva, Christof Monz, Matteo Negri, Aurélie Névéol, Mariana Neves, Martin Popel, Matt Post, Raphael Rubino, Carolina Scarton, Lucia Specia, Marco Turchi, Karin Verspoor, and Marcos Zampieri. 2016. Findings of the 2016 conference on machine translation. In Proceedings of the First Conference on Machine Translation: Volume 2, Shared Task Papers, pages 131-198, Berlin, Germany. Association for Computational Linguistics.

Ondřej Bojar, Rajen Chatterjee, Christian Federmann, Barry Haddow, Matthias Huck, Chris Hokamp, Philipp Koehn, Varvara Logacheva, Christof Monz, Matteo Negri, Matt Post, Carolina Scarton, Lucia Specia, and Marco Turchi. 2015. Findings of the 2015 workshop on statistical machine translation. In Proceedings of the Tenth Workshop on Statistical Machine Translation, pages 1-46, Lisbon, Portugal. Association for Computational Linguistics.

Ondřej Bojar, Christian Federmann, Mark Fishel, Yvette Graham, Barry Haddow, Philipp Koehn, and Christof Monz. 2018. Findings of the 2018 conference on machine translation (WMT18). In Proceedings of the Third Conference on Machine Translation: Shared Task Papers, pages 272-303, Belgium, Brussels. Association for Computational Linguistics.

Miquel Esplà, Mikel Forcada, Gema Ramírez-Sánchez, and Hieu Hoang. 2019. ParaCrawl: Web-scale parallel corpora for the languages of the EU. In Proceedings of Machine Translation Summit XVII Volume 2: Translator, Project and User Tracks, pages 118-119, Dublin, Ireland. European Association for Machine Translation.

Mozhdeh Gheini and Jonathan May. 2019. A universal parent model for low-resource neural machine translation transfer. arXiv preprint arXiv:1909.06516.

Thamme Gowda and Jonathan May. 2020. Finding the optimal vocabulary size for neural machine translation. In Findings of the Association for Computational Linguistics: EMNLP 2020, pages 3955-3964, Online. Association for Computational Linguistics.

Barry Haddow and Faheem Kirefu. 2020. Pmindia - a collection of parallel corpora of languages of india.

Charles R. Harris, K. Jarrod Millman, St'efan J. van der Walt, Ralf Gommers, Pauli Virtanen, David Cournapeau, Eric Wieser, Julian Taylor, Sebastian Berg, Nathaniel J. Smith, Robert Kern, Matti Picus, Stephan Hoyer, Marten H. van Kerkwijk, Matthew Brett, Allan Haldane, Jaime Fern'andez del R'10, Mark Wiebe, Pearu Peterson, Pierre G'erardMarchant, Kevin Sheppard, Tyler Reddy, Warren 
Weckesser, Hameer Abbasi, Christoph Gohlke, and Travis E. Oliphant. 2020. Array programming with NumPy. Nature, 585(7825):357-362.

Felix Hieber, Tobias Domhan, Michael Denkowski, and David Vilar. 2020. Sockeye 2: A toolkit for neural machine translation. In Proceedings of the 22nd Annual Conference of the European Association for Machine Translation, pages 457-458, Lisboa, Portugal. European Association for Machine Translation.

Melvin Johnson, Mike Schuster, Quoc V. Le, Maxim Krikun, Yonghui Wu, Zhifeng Chen, Nikhil Thorat, Fernanda Viégas, Martin Wattenberg, Greg Corrado, Macduff Hughes, and Jeffrey Dean. 2017. Google's multilingual neural machine translation system: Enabling zero-shot translation. Transactions of the Association for Computational Linguistics, 5:339-351.

Marcin Junczys-Dowmunt, Roman Grundkiewicz, Tomasz Dwojak, Hieu Hoang, Kenneth Heafield, Tom Neckermann, Frank Seide, Ulrich Germann, Alham Fikri Aji, Nikolay Bogoychev, André F. T. Martins, and Alexandra Birch. 2018. Marian: Fast neural machine translation in $\mathrm{C}++$. In Proceedings of ACL 2018, System Demonstrations, pages 116 121, Melbourne, Australia. Association for Computational Linguistics.

Diederik P Kingma and Jimmy Ba. 2014. Adam: A method for stochastic optimization. arXiv preprint arXiv:1412.6980.

Guillaume Klein, François Hernandez, Vincent Nguyen, and Jean Senellart. 2020. The OpenNMT neural machine translation toolkit: 2020 edition. In Proceedings of the 14th Conference of the Association for Machine Translation in the Americas (Volume 1: Research Track), pages 102-109, Virtual. Association for Machine Translation in the Americas.

Guillaume Klein, Yoon Kim, Yuntian Deng, Jean Senellart, and Alexander Rush. 2017. OpenNMT: Opensource toolkit for neural machine translation. In Proceedings of ACL 2017, System Demonstrations, pages 67-72, Vancouver, Canada. Association for Computational Linguistics.

Philipp Koehn. 2005. Europarl: A parallel corpus for statistical machine translation. Proc. 10th Machine Translation Summit (MT Summit), 2005, pages 7986.

Julia Kreutzer, Jasmijn Bastings, and Stefan Riezler. 2019. Joey NMT: A minimalist NMT toolkit for novices. In Proceedings of the 2019 Conference on Empirical Methods in Natural Language Processing and the 9th International Joint Conference on Natural Language Processing (EMNLP-IJCNLP): System Demonstrations, pages 109-114, Hong Kong, China. Association for Computational Linguistics.

Taku Kudo. 2018. Subword regularization: Improving neural network translation models with multiple subword candidates. In Proceedings of the 56th Annual
Meeting of the Association for Computational Linguistics (Volume 1: Long Papers), pages 66-75, Melbourne, Australia. Association for Computational Linguistics.

Taku Kudo and John Richardson. 2018. SentencePiece: A simple and language independent subword tokenizer and detokenizer for neural text processing. In Proceedings of the 2018 Conference on Empirical Methods in Natural Language Processing: System Demonstrations, pages 66-71, Brussels, Belgium. Association for Computational Linguistics.

Anoop Kunchukuttan, Pratik Mehta, and Pushpak Bhattacharyya. 2018. The IIT Bombay English-Hindi parallel corpus. In Proceedings of the Eleventh International Conference on Language Resources and Evaluation (LREC 2018), Miyazaki, Japan. European Language Resources Association (ELRA).

Thang Luong, Hieu Pham, and Christopher D. Manning. 2015. Effective approaches to attention-based neural machine translation. In Proceedings of the 2015 Conference on Empirical Methods in Natural Language Processing, pages 1412-1421, Lisbon, Portugal. Association for Computational Linguistics.

Chris Mattmann and Jukka Zitting. 2011. Tika in action.

Graham Neubig. 2011. The Kyoto free translation task. http://www.phontron.com/kftt.

Graham Neubig and Junjie Hu. 2018. Rapid adaptation of neural machine translation to new languages. In Proceedings of the 2018 Conference on Empirical Methods in Natural Language Processing, pages 875-880, Brussels, Belgium. Association for Computational Linguistics.

Graham Neubig, Matthias Sperber, Xinyi Wang, Matthieu Felix, Austin Matthews, Sarguna Padmanabhan, Ye Qi, Devendra Sachan, Philip Arthur, Pierre Godard, John Hewitt, Rachid Riad, and Liming Wang. 2018. XNMT: The eXtensible neural machine translation toolkit. In Proceedings of the 13th Conference of the Association for Machine Translation in the Americas (Volume 1: Research Track), pages 185-192, Boston, MA. Association for Machine Translation in the Americas.

Myle Ott, Sergey Edunov, Alexei Baevski, Angela Fan, Sam Gross, Nathan Ng, David Grangier, and Michael Auli. 2019. fairseq: A fast, extensible toolkit for sequence modeling. In Proceedings of the 2019 Conference of the North American Chapter of the Association for Computational Linguistics (Demonstrations), pages 48-53, Minneapolis, Minnesota. Association for Computational Linguistics.

Kishore Papineni, Salim Roukos, Todd Ward, and WeiJing Zhu. 2002. Bleu: a method for automatic evaluation of machine translation. In Proceedings of the 40th Annual Meeting of the Association for Computational Linguistics, pages 311-318, Philadelphia, 
Pennsylvania, USA. Association for Computational Linguistics.

Adam Paszke, Sam Gross, Francisco Massa, Adam Lerer, James Bradbury, Gregory Chanan, Trevor Killeen, Zeming Lin, Natalia Gimelshein, Luca Antiga, Alban Desmaison, Andreas Kopf, Edward Yang, Zachary DeVito, Martin Raison, Alykhan Tejani, Sasank Chilamkurthy, Benoit Steiner, Lu Fang, Junjie Bai, and Soumith Chintala. 2019. Pytorch: An imperative style, high-performance deep learning library. In H. Wallach, H. Larochelle, A. Beygelzimer, F. d'Alché-Buc, E. Fox, and R. Garnett, editors, Advances in Neural Information Processing Systems 32, pages 8024-8035. Curran Associates, Inc.

Matt Post. 2018. A call for clarity in reporting BLEU scores. In Proceedings of the Third Conference on Machine Translation: Research Papers, pages 186191, Brussels, Belgium. Association for Computational Linguistics.

Matt Post, Chris Callison-Burch, and Miles Osborne. 2012. Constructing parallel corpora for six Indian languages via crowdsourcing. In Proceedings of the Seventh Workshop on Statistical Machine Translation, pages 401-409, Montréal, Canada. Association for Computational Linguistics.

Ofir Press and Lior Wolf. 2017. Using the output embedding to improve language models. In Proceedings of the 15th Conference of the European Chapter of the Association for Computational Linguistics: Volume 2, Short Papers, pages 157-163, Valencia, Spain. Association for Computational Linguistics.

Ivan Provilkov, Dmitrii Emelianenko, and Elena Voita. 2020. BPE-dropout: Simple and effective subword regularization. In Proceedings of the 58th Annual Meeting of the Association for Computational Linguistics, pages 1882-1892, Online. Association for Computational Linguistics.

Ye Qi, Devendra Sachan, Matthieu Felix, Sarguna Padmanabhan, and Graham Neubig. 2018. When and why are pre-trained word embeddings useful for neural machine translation? In Proceedings of the 2018 Conference of the North American Chapter of the Association for Computational Linguistics: Human Language Technologies, Volume 2 (Short Papers), pages 529-535, New Orleans, Louisiana. Association for Computational Linguistics.

Roberts Rozis and Raivis Skadinšs. 2017. Tilde MODEL - multilingual open data for EU languages. In Proceedings of the 21st Nordic Conference on Computational Linguistics, pages 263-265, Gothenburg, Sweden. Association for Computational Linguistics.

Alexander Rush. 2018. The annotated transformer. In Proceedings of Workshop for NLP Open Source Software (NLP-OSS), pages 52-60, Melbourne, Australia. Association for Computational Linguistics.
Holger Schwenk, Vishrav Chaudhary, Shuo Sun, Hongyu Gong, and Francisco Guzmán. 2019. Wikimatrix: Mining $135 \mathrm{~m}$ parallel sentences in 1620 language pairs from wikipedia. CoRR, abs/1907.05791.

Rico Sennrich, Orhan Firat, Kyunghyun Cho, Alexandra Birch, Barry Haddow, Julian Hitschler, Marcin Junczys-Dowmunt, Samuel Läubli, Antonio Valerio Miceli Barone, Jozef Mokry, and Maria Nădejde. 2017. Nematus: a toolkit for neural machine translation. In Proceedings of the Software Demonstrations of the 15th Conference of the European Chapter of the Association for Computational Linguistics, pages 65-68, Valencia, Spain. Association for Computational Linguistics.

Rico Sennrich, Barry Haddow, and Alexandra Birch. 2016. Neural machine translation of rare words with subword units. In Proceedings of the 54th Annual Meeting of the Association for Computational Linguistics (Volume 1: Long Papers), pages 17151725, Berlin, Germany. Association for Computational Linguistics.

Jörg Tiedemann. 2012. Parallel data, tools and interfaces in OPUS. In Proceedings of the Eighth International Conference on Language Resources and Evaluation (LREC'12), pages 2214-2218, Istanbul, Turkey. European Language Resources Association (ELRA).

Jörg Tiedemann. 2020. The tatoeba translation challenge - realistic data sets for low resource and multilingual MT. In Proceedings of the Fifth Conference on Machine Translation, pages 1174-1182, Online. Association for Computational Linguistics.

Ashish Vaswani, Samy Bengio, Eugene Brevdo, Francois Chollet, Aidan Gomez, Stephan Gouws, Llion Jones, Łukasz Kaiser, Nal Kalchbrenner, Niki Parmar, Ryan Sepassi, Noam Shazeer, and Jakob Uszkoreit. 2018. Tensor2Tensor for neural machine translation. In Proceedings of the 13th Conference of the Association for Machine Translation in the Americas (Volume 1: Research Track), pages 193-199, Boston, MA. Association for Machine Translation in the Americas.

Ashish Vaswani, Noam Shazeer, Niki Parmar, Jakob Uszkoreit, Llion Jones, Aidan N Gomez, Łukasz Kaiser, and Illia Polosukhin. 2017. Attention is all you need. In Advances in neural information processing systems, pages 5998-6008.

Xinyi Wang, Yulia Tsvetkov, and Graham Neubig. 2020. Balancing training for multilingual neural machine translation. In Proceedings of the 58th Annual Meeting of the Association for Computational Linguistics, pages 8526-8537, Online. Association for Computational Linguistics.

Thomas Wolf, Lysandre Debut, Victor Sanh, Julien Chaumond, Clement Delangue, Anthony Moi, Pierric Cistac, Tim Rault, Remi Louf, Morgan Funtowicz, Joe Davison, Sam Shleifer, Patrick von Platen, 
Clara Ma, Yacine Jernite, Julien Plu, Canwen Xu, Teven Le Scao, Sylvain Gugger, Mariama Drame, Quentin Lhoest, and Alexander Rush. 2020. Transformers: State-of-the-art natural language processing. In Proceedings of the 2020 Conference on Empirical Methods in Natural Language Processing: System Demonstrations, pages 38-45, Online. Association for Computational Linguistics.

Yonghui $\mathrm{Wu}$, Mike Schuster, Zhifeng Chen, Quoc V. Le, Mohammad Norouzi, Wolfgang Macherey, Maxim Krikun, Yuan Cao, Qin Gao, Klaus Macherey, Jeff Klingner, Apurva Shah, Melvin Johnson, Xiaobing Liu, Lukasz Kaiser, Stephan Gouws, Yoshikiyo Kato, Taku Kudo, Hideto Kazawa, Keith Stevens, George Kurian, Nishant Patil, Wei Wang, Cliff Young, Jason Smith, Jason Riesa, Alex Rudnick, Oriol Vinyals, Greg Corrado, Macduff Hughes, and Jeffrey Dean. 2016. Google's neural machine translation system: Bridging the gap between human and machine translation. CoRR, abs/1609.08144.

Matei Zaharia, Reynold S Xin, Patrick Wendell, Tathagata Das, Michael Armbrust, Ankur Dave, Xiangrui Meng, Josh Rosen, Shivaram Venkataraman, Michael J Franklin, et al. 2016. Apache spark: a unified engine for big data processing. Communications of the ACM, 59(11):56-65.

Biao Zhang, Philip Williams, Ivan Titov, and Rico Sennrich. 2020. Improving massively multilingual neural machine translation and zero-shot translation. In Proceedings of the 58th Annual Meeting of the Association for Computational Linguistics, pages 16281639, Online. Association for Computational Linguistics.

Michał Ziemski, Marcin Junczys-Dowmunt, and Bruno Pouliquen. 2016. The united nations parallel corpus v1.0. In Proceedings of the Tenth International Conference on Language Resources and Evaluation (LREC'16), pages 3530-3534, Portorož, Slovenia. European Language Resources Association (ELRA).

Barret Zoph, Deniz Yuret, Jonathan May, and Kevin Knight. 2016. Transfer learning for low-resource neural machine translation. In Proceedings of the 2016 Conference on Empirical Methods in Natural Language Processing, pages 1568-1575, Austin, Texas. Association for Computational Linguistics. 